\title{
Lo que nos dio y no nos dio Bourbaki*
}

(What Bourbaki Has and Has Not Given Us)

\author{
Enetz Ezenarro Arriola
}

Received: 22/10/2015

Final Version: 12/04/2016

BIBLID 0495-4548(2017)32:1p.25-40

DOI: $10.1387 /$ theoria. 15199

RESUMEN: Bourbaki nos enseñó el potencial que guarda el concepto de estructura matemática para reorganizar, sistematizar y unificar el entramado matemático. Pero la evolución de la matemática, en las últimas décadas deja patente las limitaciones de este enfoque. En este artículo analizamos las contribuciones de Bourbaki a lo que denominamos fundamentación 'interna' de la matemática y señalamos, a su vez, las que a nuestro juicio son sus principales carencias. A continuación bosquejamos brevemente algunas evidencias sobre las que sustentamos la perspectiva denominada funcionalismo estructuralista. Según ésta, la noción general de morfismo caracteriza la naturaleza dinámica de la matemática actual.

Palabras clave: Bourbaki, estructuralismo, fundamentos internos de la matemática, funcionalismo estructuralista.

ABSTRACT: Bourbaki showed us the potential inherent within the concept of mathematical structure for re-organizing, systematically arranging and unifying the mathematical framework. But mathematics' development in recent decades has flagged up the limitations of this approach. In this article we analyse Bourbaki's contributions to what we term the "internal" foundations of mathematics, and at the same time we indicate where, in our view, they fall short. We go on to outline some of the evidence on which we base the viewpoint termed structural functionalism. According to the latter, the general notion of morphism characterizes the dynamic nature of present-day mathematics.

Keywords: Bourbaki, structuralism, internal foundations of mathematics, structuralist functionalism.

\section{Introducción: Bourbaki, el nuevo Euclides}

El objetivo, tanto de Euclides como de Bourbaki, era presentar una exposición lo más clara y coherente posible de las matemáticas de su tiempo. Su trabajo se podría considerar como un trabajo de reorganización de las matemáticas, cuestión ésta que guarda desde siempre una íntima relación con el ámbito de los fundamentos de las matemáticas. Bien es cierto que clásicamente existe la tendencia de limitar el campo de la fundamentación de las matemáticas a empresas que desde fuera, es decir, obedeciendo a intereses más filosóficos que puramente matemáticos, pretendían dotar a las matemáticas de unos esquemas de seguridad sobre los cuales se sostuviese el 'edificio matemático'. Nos referimos sobre todo al debate lógico-filosófico que surge a finales del siglo XIX (logicismo, formalismo, intuicionismo), como continuación del proceso de rigorización y aritmetización del análisis. Como es sabido, este debate

* Quisiera agradecer a Jesus Mari Larrazabal y a dos evaluadores anónimos por los comentarios realizados sobre versiones previas de este artículo que han contribuido notablemente a su mejora. 
pierde gran parte de su relevancia con los resultados de limitación que Gödel demostró desde la metamatemática, estableciendo la imposibilidad de encontrar esas bases seguras fuera de las matemáticas. Ante esta vía cerrada, reivindicamos como quehacer propio del ámbito de la fundamentación en las matemáticas una vía que calificamos como de fundamentación interna. Son claros exponentes de esta vía, precisamente, los trabajos de Euclides y Bourbaki, o también los procesos de rigorización y aritmetización que se dieron durante los siglos XVIII y XIX, y que transformaron el Cálculo Infinitesimal en Análisis Matemático. Todos ellos son procesos de rigorización, organización o generalización, que se incluyen de forma legítima dentro del propio funcionamiento de la disciplina. No en vano los intentos de comprender mejor las diferentes teorías y de establecer relaciones fundamentales entre ellas, han sido desde siempre un importante motor para la evolución de las matemáticas (Mac Lane, 1986).

Nicolas Bourbaki es conocido hoy en día principalmente por su obra Éléments de mathématique (Bourbaki, 1939-). Como se puede observar el nombre de la obra contiene una clara referencia a los Elementos de Euclides, contribución que fue realizada con más de 2000 años de antelación. Esto de ningún modo es fruto de una casualidad. Bourbaki pretendía dejar patente a través del título la conexión existente entre el principal objetivo que perseguía la obra clásica de Euclides y la que él mismo se disponía a emprender (Hernández, 2002). Un objetivo que no era otro que reorganizar la matemática de una forma sistemática, poniendo un especial énfasis en la clarificación y unificación del entramado axiomático mediante el cual se interrelacionaban las diferentes proposiciones, logrando de esta forma integrar en un mismo cuerpo partes que previamente parecían estar muy distantes unas de otras. En este sentido el trabajo de Euclides, aunque todavía no totalmente riguroso desde un punto de vista moderno, es un claro referente de lo que se ha llamado un sistema axiomático-deductivo.

Dada una teoría su reformulación a través del método axiomático-deductivo permite esclarecer cuáles son sus elementos básicos. Es decir, seleccionar las proposiciones primitivas de la teoría desde un punto de vista lógico, de modo que a partir de ellas sea posible deducir una tras otra todas las demás. En este caso a las proposiciones primitivas seleccionadas se les llamará axiomas o bien postulados de la teoría. Una condición que se le puede exigir al conjunto de axiomas es que sean lógicamente independientes entre sí; que no haya forma de deducir ninguna de ellas a partir de las restantes. En algunas ocasiones, en la práctica, esta condición es obviada en beneficio de la intuición ${ }^{1}$. El proceso de axiomatización de una teoría puede llevarse a cabo de diferentes formas. Puede haber más de una posible axiomatización de una misma teoría. Uno de los principales criterios a la hora de evaluar la eficacia de una axiomatización es la de la inteligibilidad de la que ésta dota a la teoría: una buena selección de axiomas debería dar lugar a una estructuración lo más simple posible, sin perder de vista la eficacia operativa de sus aplicaciones y, por ende, permitiría una comprensión más profunda de la teoría.

Debido a su potencial clarificador, ésta era la metodología que priorizaba Hilbert, a la hora de exponer de una forma concluyente una teoría culminada en la que la mayoría de las preguntas habían sido respondidas (Corry, 1996). Tanto para Euclides como para Bourbaki la inteligibilidad profunda de los resultados matemáticos es posible, precisamente, a través de la utilización de la herramienta axiomática (Bourbaki, 1950).

1 Cabría recordar en este punto el grado de abstracción que adopta, por ejemplo, el sistema axiomático, ahora sí totalmente riguroso, que dio Hilbert para la geometría euclidea (Hilbert, 1899), demostrando a su vez la independencia lógica del sistema. 
Podría decirse que tanto el concepto clásico de 'edificio matemático' que se deriva de la imagen de las matemáticas establecidas por Euclides como su extensión moderna de 'ciudad matemática' propuesta por Bourbaki (1950) para adaptar la metáfora arquitectónica al complicado panorama del siglo xx, están íntimamente ligados a la amplia utilización en la matemática de la metodología axiomática ${ }^{2}$. En el primero de los casos se sobreentiende que siempre que somos capaces de dar una axiomatización de cierta teoría, es posible considerar esta teoría como un edificio que se sustenta lógicamente sobre los fundamentos que ofrecen los axiomas. La metáfora del edificio sirve para representar la unidad de la teoría: cada proposición está relacionada con cualquier otra mediante las conexiones lógicas que se derivan de los fundamentos; y además dicha relación puede hacerse totalmente explícita.

Existe, sin embargo, un concepto fundamental que es explotado sistemáticamente por Bourbaki hasta el punto de considerarlo eje central de su propuesta de reorganización, y al cual, por razones obvias, Euclides no tuvo acceso. Es éste el concepto de las estructuras matemáticas, a través de cuyas combinaciones es posible, según Bourbaki, reconstruir en su forma más profunda las teorías matemáticas y sus interrelaciones, evolucionando hacia un panorama matemático cada vez más fundamental e inteligible. Estas consideraciones son las que dan pie a la mencionada metáfora de las matemáticas como ciudad en constante reordenación que citamos a continuación ${ }^{3}$ :

Es como una gran ciudad, cuyos barrios periféricos y suburbios invaden incesantemente, y de una forma un tanto caótica, el terreno circundante, mientras el centro se reconstruye cada cierto tiempo, cada vez de acuerdo con un plan concebido de forma más clara y un orden más majestuoso, derruyendo las partes viejas con sus laberintos de callejuelas, y proyectando hacia la periferia nuevas avenidas, más directas, amplias y más confortables.

\section{Elálgebra moderna}

Como en el caso de muchos otros conceptos importantes en matemáticas, es difícil seguir la pista de los diferentes componentes que desembocaron en la génesis de las estructuras matemáticas ${ }^{4}$. Bien es cierto, de todas formas, que podríamos establecer un

2 Ferreirós y Gray (2006) ofrecen en la introducción de su libro un breve repaso de la evolución de la metáfora arquitectónica de las matemáticas.

3 It is like a big city, whose outlying districts and suburbs encroach incessantly, and in a somewhat chaotic manner, on the surrounding country, while the center is rebuilt from time to time, each time in accordance with a more clearly conceived plan and a more majestic order, tearing down the old sections with their labyrinths of alleys, and projecting towards the periphery new avenues, more direct, broader and more commodious (Bourbaki, 1950, p. 230). Traducción del autor.

4 No es mi intención en este artículo repasar la evolución del estructuralismo en matemáticas. Simplemente pretendo dar unas pinceladas y señalar algunos autores que tuvieron una influencia directa en el punto de partida del grupo Bourbaki. Es por esto que no hago ninguna referencia a autores tan trascendentales para el desarrollo de esta concepción de las matemáticas como pueden ser Grassman, Hilbert o Hausdorff entre otros muchos. El lector interesado podrá encontrar una exposición sucinta de los principales hitos del desarrollo de las matemáticas sobre los que se sustenta esta visión de las matemáticas en (Dieudonné, 1979). Con más detenimiento (Corry, 1996) estudia la cuestión de la concepción estructuralista de las matemáticas proveniente principalmente de la eficaz sistematización del álgebra estructuralista moderna. 
claro límite en esta evolución en los trabajos realizados por Galois en la primera parte del siglo XIX. Él fue uno de los primeros en hacer uso explícito de estructuras matemáticas, en su caso la de grupo y también indirectamente la de cuerpo, para resolver de la forma más general y más simple posible el problema de la existencia de soluciones mediante radicales de ecuaciones polinómicas (Wussing, 1969). Y aunque la comunidad matemática necesitara mucho tiempo para poder asimilar el verdadero significado de sus trabajos, el método utilizado por Galois resultó ser revolucionario y transformó profundamente las matemáticas, suponiendo uno de los avances que mayor repercusión han tenido en su evolución, equiparable a la introducción de coordenadas por Fermat y Descartes en el siglo XVII o a la posterior invención del cálculo por Newton y Leibniz. Con Galois se precipita de forma totalmente inesperada el paso de un estadio a otro en la evolución interna de las matemáticas (Cartier, 1998). Sin duda alguna sus métodos se pueden considerar un verdadero paso de gigante en el camino hacia la simplificación, que siempre ha supuesto uno de los principales criterios que han determinado el devenir de las matemáticas.

En este método se recoge lo que podríamos denominar el 'principio estructuralista': tratar de caracterizar cualquier problema en función de la estructura o el ente abstracto que recoge toda la información relevante, y a ser posible nada más que eso. La perspectiva estructural de un problema permite abordarlo en su forma más general, más básica, cerrándolo con absoluta 'limpieza', y sin dejar cabos sueltos. Nos referimos, por ejemplo, a los casos en los que no encontramos otra opción que razonar subdividiendo el problema en una casuística, que da pie a una diversidad de métodos y que, en definitiva, dificultan la obtención de una concepción integral del asunto. En este sentido se han de entender las palabras de Mac Lane (Mac Lane, 1986) de que no todas las demostraciones son equivalentes. Toda demostración sirve para justificar la veracidad del teorema, sin embargo, algunas, y en especial las que inciden en la estructura interna del problema, si es que es posible hallarla, son las que mejor explican el 'fenómeno' en cuestión. Es por ello deseable no conformarse con cualquier demostración y perseverar hasta encontrar una que explique por qué es cierto eso que se asevera. El principio estructuralista al que nos referimos con Galois estaría estrechamente relacionado con la búsqueda de la explicación profunda de las cuestiones matemáticas. En el caso particular de la búsqueda de soluciones en términos de radicales para una ecuación polinómica dada, esta explicación profunda vendría de la mano de lo que hoy en día se llama grupo de Galois de esa ecuación, que es en este caso la estructura implicada.

Es dentro del álgebra, donde surge la metodología estructuralista y es en ella donde se desarrolla y extiende primeramente la concepción de la matemática que conlleva dicho punto de vista. Una vez que se comprende el alcance de la contribución de Galois no tardan en aparecer nuevos ejemplos donde las estructuras algebraicas vuelven a jugar el mismo papel fundamental. Podríamos encontrar claros ejemplos de esta afirmación en los trabajos realizados por Dedekind en la teoría algebraica de números, generalizando la teoría de la divisibilidad de Kummer a través de la teoría de los ideales (Corry, 1996). Incluso, podemos encontrar ejemplos en contextos no-algebraicos, como puede ser el Programa de Erlangen (Klein, 1872) que Klein desarrolló para determinar el modo más simple de organizar los diferentes sistemas geométricos, basándose en las relaciones existentes entre sus 'grupos fundamentales', que suponen en cierto sentido el final de la geometría clásica y el comienzo de 
la geometría moderna 5 . Así, la proliferación de diferentes estructuras algebraicas que demuestran no solo ser una herramienta práctica a la hora de abordar problemas concretos, sino también jugar un papel simplificador, generalizador y, en definitiva, clarificador y organizador allí donde se utilizan, da pie en una segunda fase a que surja, por generalización, el concepto abstracto de estructura algebraica, que se convertirá rápidamente en el concepto vertebrador de la nueva álgebra. Las estructuras pasarán así de ser herramientas puramente heurísticas utilizadas para la resolución de problemas a ser verdaderos objetos de estudio, con el correspondiente surgimiento de las teorías abstractas de las estructuras algebraicas generales.

Este es el punto de vista que aparece expuesto por primera vez en Moderne Algebra (van der Waerden, 1930-1931) y que van der Waerden fue adquiriendo y madurando en gran medida a través de su contacto tanto con Emil Artin como con Emmy Noether, los dos algebristas más eminentes del momento. Aunque bien es cierto que es a van der Waerden a quien se le debe el mérito de organizar y adoptar aquella exposición concreta de la teoría de las estructuras algebraicas, no es menos cierto que los grandes desarolladores de los conceptos y resultados estructurales que exponía van der Waerden fueron, precisamente, Artin y Noether, junto con la labor previa de Dedekind (Corry, 1996).

Según la novedosa visión del álgebra que ofrece van der Waerden, el álgebra es el campo donde se estudian las propiedades de las diferentes estructuras algebraicas en su forma más general y que resultan de interés en muy diversas áreas tanto de las propias matemáticas como de fuera de ellas, en especial en física. En Moderne Algebra se desarrollan sistemáticamente las teorías axiomáticas de grupos, anillos, cuerpos, módulos, espacios vectoriales, etc. Se podría decir, utilizando una terminología muy utilizada en la biología que todas ellas no son más que diferentes especies dentro de un mismo género: el de las estructuras algebraicas. En van der Waerden no podemos encontrar más que informalmente el concepto abstracto de estructura algebraica. Esta se percibe de forma implícita pero contundente en la forma en que se desarrollan las diferentes teorías axiomáticas de las diferentes estructuras. En todas hallamos construcciones, cuestiones y resultados homólogos, en la medida en que cada estructura lo permita. Y, en cambio, problemas que previamente se consideraban centrales en álgebra, como la resolución de ecuaciones polinómicas son relegados a meras aplicaciones de principios más generales obtenidos dentro de las teorías generales de las estructuras algebraicas. Esta es la imagen del álgebra que se impondrá a partir de ese momento, ya que, sin lugar a dudas el principio ordenador que ofrece el concepto general de estructura algebraica ${ }^{6}$, tiene un alcance superior y dota al álgebra de una unidad y una coherencia que nunca antes había conocido, integrando, relacionando y generalizando resultados clásicos. Podría decirse, en definitiva, que la introducción de la perspectiva estructuralista, produjo un cambio profundo en la concepción y estructuración del álgebra (Corry, 1996).

5 Nos referimos a que la perspectiva de la geometría cambia profundamente con Klein, que generaliza la concepción de la propia geometría. En términos modernos podríamos decir que una geometría se compone básicamente de un grupo de Lie $G$, de una variedad diferenciable $S$ y de una acción del grupo $G$ sobre $S$, que es diferenciable, transitiva y efectiva (Sharpe, 1997). Esto nos permite centrar la atención sobre el grupo $G$, en vez de sobre la variedad $S$, ofreciendo un punto de vista algebraico de la geometría.

6 Podríamos definir genéricamente una estructura algebraica como un conjunto no vacío dotado de diversas (una o más) leyes de composición internas y/o externas, que satisfacen una serie de propiedades, que son las que definen la estructura. 
Está claro que la gran aportación de van der Waerden corresponde a los fundamentos del álgebra más que al álgebra en sí misma. No hay nuevos teoremas en su obra (que no sean generalizaciones y reinterpretaciones más o menos directas de resultados previamente conocidos). Su gran aportación está relacionada con la forma de exponer la teoría, ofreciendo un contexto homogéneo de forma que resultados conocidos con antelación, adquiriesen, sin embargo, una organización y una exposición más simple y unificada.

\section{3. Éléments de mathématique (1939-)}

Es precisamente del asombro que causa el inmenso potencial del álgebra moderna (estructuralista) según se presenta en la obra de van der Waerden, de donde parte el proyecto Bourbakista (Dieudonné, 1970, p. 137). Bourbaki vio la posibilidad de extender el concepto general de estructura algebraica a otro, más general aún, de estructura matemática, de forma que este concepto que había demostrado ser fundamental en la clarificación del álgebra, guiase una reorganización, semejante de manera global, en toda la matemática (pura ${ }^{7}$ ). Mediante este proceso de 'algebraización' de la matemática se pretendía dotar a otros campos de una organización más racional, y de la misma claridad con la que se había provisto el álgebra, en su tránsito del álgebra clásica al álgebra moderna. Así, de la misma forma en que podíamos decir que el álgebra se ocupaba de estudiar las estructuras algebraicas, la tesis fundamental del proyecto bourbakista es sobre la extensión de esta concepción a toda la matemática, sosteniendo que la matemática es la ciencia que estudia las estructuras y que la labor de los matemáticos, es por tanto, hallar las estructuras que subyacen a los problemas matemáticos, con la intención de estudiarlos en su forma más general, de manera que no solo tengan aplicación inmediata en el mismo problema en el que surgen, sino, más allá de éste, en otros que, aunque diferentes en la superficie compartan la estructura interna. De ahí la importancia de que las teorías sean formuladas en los términos más generales posibles: un único teorema sirve de este modo para todo un abanico de aplicaciones, una vez contextualizado; una vez interpretado. Esta es la posición que venimos a llamar estructuralista, en el ámbito de la fundamentación interna de las matemáticas. Merece la pena recordar en este punto las palabras de Dieudonné sobre el trabajo de Bourbaki ${ }^{8}$ :

7 El interés de Bourbaki se centra única y exclusivamente en la matemática pura en contraposición con la matemática aplicada. Por señalar simplemente un ejemplo, a pesar del auge que vivió la Teoría de Probabilidades durante el siglo xx, en especial en los años en que echó a andar Bourbaki, ésta no tuvo mención en su obra pese a que algunos integrantes del grupo sí que se interesaron a nivel personal (Schwartz, 1997).

8 Bourbaki sets off, if you like, from a basic belief, an unprovable metaphysical belief we willingly admit. It is that mathematics is fundamentally simple and that for each mathematical question there is, among all the possible ways of dealing with it, a best way. We can give examples where this is true and examples where we cannot say, because up to now we have not found the optimal method. I cited, for example, group theory and analytical number theory, which are characteristic. In both one has a quantity of methods, each one more clever than the last. This is splendid and ingenious and of a complexity never before known, but we are sure that this is not the final way to deal with the question. On the other hand, take algebraic number theory. Since Hilbert, it is so systematized that we know there is a right way to handle its questions. We change them sometimes, but in the end, little by little, we manage to find one way which is better than the others. This is only a belief, I repeat, a metaphysical belief (Dieudonné, 1970, p. 145). Traducción del autor. 
Si os parece, Bourbaki parte desde una creencia básica, una creencia metafísica indemostrable que admitimos de buen grado. Se trata de que la matemática es fundamentalmente simple y que para cada cuestión matemática existe, entre las distintas formas posibles de abordarla, una que es la mejor. Podemos dar ejemplos en los cuales esto es cierto y ejemplos en los que no podemos decir nada, puesto que no hemos hallado el método óptimo hasta el momento. Citaba, por ejemplo, la teoría de grupos y la teoría analítica de números, que son característicos. En ambos uno tiene una cantidad de métodos, cada cual más agudo. Esto es magnífico e ingenioso y de una complejidad nunca antes conocida, pero estamos seguros de que no es la forma definitiva de tratar la cuestión. Por otro lado, tomemos la teoría algebraica de números. Desde Hilbert, está tan sistematizada que sabemos que hay una forma correcta de tratar sus cuestiones. A veces las cambiamos, pero al final, poco a poco, nos las arreglamos para encontrar una forma que es mejor que las demás. Esto es tan solo una creencia, lo repito, una creencia metafísica.

Bourbaki estaba convencido de que la perspectiva que van der Waerden ofreció del álgebra se podía generalizar a toda la matemática, y que la dificultad radicaba en hallar para cada teoría la estructura idónea, de la misma forma que había hecho Galois con la teoría de las ecuaciones algebraicas, o Klein con los diferentes sistemas geométricos. Y, como bien recordaba Dieudonné, era ésta una convicción indemostrable, no se trataba de una proposición matemática sino de una fuerte creencia basada en la experiencia de lo que había sucedido en el álgebra.

Extrapolando el trabajo realizado por van der Waerden dentro del campo del álgebra, Bourbaki adoptó el concepto de estructura matemática como principio organizativo fundamental de las matemáticas. Pero en este caso el concepto de estructura debía ser más general que el concepto de estructura algebraica, para poder abrirse a todos los ámbitos de la matemática del momento. Surge así el concepto general de estructura matemática, piedra angular del proyecto bourbakista.

Las estructuras matemáticas de Bourbaki son ante todo conjuntos 'estructurados' o dotados de 'estructura'. Cabría mencionar, quizá, que si bien van der Waerden también había elegido el lenguaje conjuntista como herramienta básica para el desarrollo de su exposición, el trabajo de Bourbaki es mucho más riguroso: es su intención prescindir en todo momento de cualquier apelación a cualquier intuición física o espacial y para ello adopta un estilo extremadamente lineal, según el cual, cualquier resultado se deriva de una combinación concreta de los axiomas y los resultados previos mediante reglas deductivas previamente establecidas. Así pretende dotar a las matemáticas de un fundamento explícito, sobre el cual erigirá con sumo rigor el pretendido cuerpo deductivo de las matemáticas. Bourbaki no duda en señalar a la teoría de conjuntos como la única fundamentación posible de las matemáticas. Su interés es únicamente matemático y no pretende dotar al edificio matemático de una seguridad total que ni busca, ni cree necesaria. Esto era así especialmente a la vista de los teoremas de limitación de Gödel, que parecían descartar toda posibilidad de dotar a las matemáticas de esquemas de seguridad, que evitasen cualquier aparición de contradicciones del tipo russelliano en el futuro. Bourbaki busca simplemente delimitar un campo de juego lo suficientemente amplio como para poder desarrollar los razonamientos matemáticos clásicos (Dieudonné, 1970), sin que le preocupen demasiado los problemas lógico-filosóficos relacionados con lo que Davis y Hersh (1981) denominaban el 'mito euclídeo'. La teoría

9 Según este mito las proposiciones matemáticas serían verdaderas, objetivas y eternas y las matemáticas constituirían una realidad platónica extratemporal y extrasensorial. Es esta una idea que ha tenido grandes repercusiones filosóficas a lo largo de la historia, y que aún hoy en día sigue vigente, incluso en 
de conjuntos axiomática ofrece las bases suficientemente seguras (aunque no totalmente seguras) para la práctica matemática clásica. En el primer volumen Théorie des ensembles, Bourbaki nos ofrece su propia versión de una teoría axiomática de conjuntos, similar a la que dio Zermelo en $1908^{10}$.

Bourbaki pretende, en su afán de adoptar el concepto de estructura como eje articulador de las teorías matemáticas, reorganizar la matemática pura valiéndose de una jerarquía de estructuras. En la base de esta jerarquía se encuentran las estructuras más generales y a la vez más simples (con un menor número de condiciones). Son las llamadas 'estructuras madre', cuyas diferentes combinaciones dan lugar a un sinfín de estructuras, más concretas y a su vez más complejas, que combinan las propiedades de las estructuras madres que la definen. Las estructuras madre pueden ser de tres tipos (a la espera de que la propia evolución de las matemáticas pueda producir nuevas estructuras madre y, por ende, más estructuras derivadas): las estructuras algebraicas, las estructuras de orden y las estructuras topológicas. Las estructuras algebraicas están formadas por conjuntos dotados de diferentes leyes de composición, que pueden ser internas (operaciones), o externas (operadores), y vienen determinadas por una serie de axiomas. Por ejemplo, un grupo $G$ es un conjunto no vacío dotado de una ley de composición interna, que satisface los tres axiomas siguientes: para todo $x, y, z$ de $G$, (G1) $x(y \times z)=(x \times \mathrm{y}) z$; (G2) existe un elemento $e$ en $G$ tal que, $x \times e=e \times x=x$; (G3) para todo $x$ en $G$ existe $x^{-1}$ en $G$ tal que, $x \times x^{-1}=\mathrm{x}^{-1} \times x=1$. Se dice en este caso que $(G, x)$ es un grupo. Las estructuras de orden son conjuntos dotados de una relación de orden parcial, es decir, relaciones que son reflexivas, antisimétricas y transitivas. Por último, las estructuras topológicas son conjuntos no vacíos $A$, en los que a cada elemento del conjunto se le asocia una colección de subconjuntos de $A$, que satisfacen una serie de axiomas (lo que se llamará un 'sistema de entornos' de ese punto) (Bourbaki, 1950).

Según Bourbaki, partiendo de esta base común, las diferentes teorías matemáticas podrían organizarse a través de una jerarquía de estructuras, más o menos sofisticadas en función del número de estructuras madre que fuese necesario combinar (de forma coherente) para poder derivar los resultados requeridos. Por ejemplo los grupos topológicos, son conjuntos en los que se define una ley de composición interna, que satisface los axiomas de grupo, y a su vez, se define para cada elemento del conjunto un sistema de entornos que dota al conjunto de estructura topológica, resultando además que tanto la operación binaria de grupo como la operación unaria de 'tomar el elemento inverso' sean continuas considerándolas como funciones entre espacios topológicos. Se ve en este ejemplo que la combinación de estructuras sobre un mismo conjunto se ha de dar de forma que ambas estructuras estén intrínsecamente relacionadas. El hecho es que las estructuras que se obtienen añadiendo condiciones a estructuras previamente establecidas, son más fructíferas pero menos aplicables en cuanto que se va perdiendo en generalidad. Son más fructíferas puesto que al satisfacerse nuevas condiciones se posibilita la obtención de resultados que serían falsos en otras circunstancias. Ahora bien, las nuevas condiciones dificultan la posibilidad de hallar modelos que las satisfagan, de forma que las teorías correspondientes a estas nuevas

gran parte de la comunidad matemática. Si bien es cierto que en la mayoría de los casos es más una cuestión de conveniencia que el producto de una reflexión filosófica sosegada sobre las matemáticas.

10 (Kneebone, 1963) describe brevemente el sistema axiomático que presentó Bourbaki como fundamento de su obra. (Anacona et al., 2014) ofrece un análisis más detallado de este mismo sistema demostrando que es equivalente al sistema ZFC sin el axioma de fundación. 
estructuras son mucho más concretas y corren el riesgo de perder toda relevancia dentro del panorama matemático.

Esta jerarquización imprime un orden natural entre las teorías más generales y las más específicas. Este ordenamiento muchas veces no guarda relación con la imagen clásica de las matemáticas. Bourbaki en su tarea de reorganización no duda en seguir los criterios que se derivan de la adopción de un punto de vista estructuralista de las matemáticas. Un claro ejemplo de este hecho lo podemos encontrar en la ubicación que encuentra el análisis real dentro de la obra de Bourbaki. Un campo de las matemáticas que desde su creación se había considerado como uno de los pilares centrales de la matemática moderna, se ve relegado, sin embargo, a una posición secundaria, debido a que la estructura matemática correspondiente es sumamente concreta: en ella se combinan las estructuras algebraicas de grupo, anillo, cuerpo y espacio vectorial, con una estructura de orden total y una estructura topológica euclidea, entre otras. La prioridad de una teoría en la obra de Bourbaki es directamente proporcional a la generalidad de la estructura en la que se enmarca. Como lo hacía notar Dieudonné en la cita que recordábamos más arriba, Bourbaki tiene la convicción 'metafísica' de que la perspectiva estructural, como ocurrió con van der Waerden, permite exponer la teoría de forma óptima, y este fin justifica la posibilidad de que campos que se han considerado fundamentales históricamente queden relegados, en la nueva exposición, a un segundo o a un tercer plano. No sería ni más ni menos que el precio que habría que pagar para poder avanzar a un estadio en el que la matemática adoptara una forma mucho más simple, coherente e inteligible.

\section{Lo que nos dio y no nos dio Bourbaki}

Si algo podemos afirmar sin ningún lugar a duda desde la perspectiva actual es que la tarea que se impuso Bourbaki no ha sido, finalmente, llevada a término. El trabajo de reconstrucción era un trabajo arduo, había que escribir y reescribir las teorías en busca de esa 'forma óptima'; en busca de la estructura que articulase de la forma más simple y elegante su exposición. Para los años noventa del siglo pasado estaba claro que el final de Bourbaki estaba cerca, ya que la rápida expansión multidireccional que se estaba dando en las matemáticas hacía que su labor fuese inconcebible en la práctica (Mashaal, 2002).

Una de las críticas más razonables que se le ha dirigido al trabajo realizado por Bourbaki es, precisamente, la que afirma que la perspectiva que éste proponía en su obra dejaba fuera muchas partes importantes de las matemáticas (Hermann, 1986; Mac Lane, 1996; Cartier, 1998), debido a que no se adecuaban (o por lo menos no se conocía la forma de hacerlo) a los criterios fundamentales que se habían adoptado de forma previa, y que constituían la esencia misma de la matemática. No está nada claro cuáles son las estructuras subyacentes a ciertas teorías, y por tanto como se podrían desarrollar sus teorías de forma estructural. Por ejemplo, como bien recuerda Mac Lane (1996), gran parte del estudio de las ecuaciones en derivadas parciales y sus soluciones no puede ser debidamente descrita en términos de estructuras axiomatizadas. Otro tanto ocurre con las funciones de una variable compleja u otras áreas del análisis moderno como pueden ser el análisis armónico, los sistemas dinámicos, el caos, los fractales, cuestiones relacionadas con la física matemática, etcétera. En general se podría decir que el análisis moderno no se integra debidamente en el trabajo de Bourbaki, salvando la teoría de funciones de una variable real y el análisis funcional, 
que sí se recogen. El análisis funcional, en particular, se ha venido en llamar informalmente 'la parte más algebraica del análisis', haciendo referencia a las posibilidades que ofrece para ser desarrollado axiomáticamente. Junto al análisis también podríamos mencionar los diferentes campos relacionados con la geometría moderna, que surgen a partir de los trabajos de Elie Cartan, incluyendo áreas hoy tan importantes como la topología algebraica y sus campos adyacentes. Todos ellos encuentran mejor acomodo dentro de la teoría de categorías de Mac Lane y Eilenberg, e incluso, en algunos casos, dentro de la teoría de topos de Grothendieck, que en cambio Bourbaki nunca hará suyas (Cartier, 1998). Sucede otro tanto con todos aquellos aspectos computacionales y algorítmicos de la matemática, que tanto han proliferado, a partir de la explosión de las ciencias de la computación como bien podrían representar la combinatoria, la teoría de grafos, etc. Por no entrar (si es que no hemos entrado ya), en la matemática aplicada, en la que Bourbaki no estaba interesado.

Merece la pena explayarse en una cuestión que acabamos de mencionar, ya que es una cuestión recurrente entre aquellos que han reflexionado sobre el valor del trabajo realizado por Bourbaki. Y no se trata de otra cosa que de la ausencia deliberada de la teoría de categorías y de topoi en los Éléments de mathématique, una vez de que estas teorías fuesen desarrolladas. Estos autores defienden que es contradictorio no hacer uso de la mejor teoría de estructuras que existía, para desarrollar el punto de vista de que la matemática es la ciencia que estudia las estructuras, que es al fin y al cabo la tesis de Bourbaki. Según ellos la teoría de categorías serviría para establecer un marco general sobre el que desarrollar la exposición de la perspectiva estructuralista de las matemáticas de forma mucho más coherente. No es esta, en cambio, la elección que hace Bourbaki. Desde el primer volumen de su obra, Théorie des ensembles, en el que fundamenta su posterior trabajo, Bourbaki abre una vía a la que posteriormente no dará ninguna continuidad por resultar demasiado oscura y difícilmente manejable en la práctica. Y es que la definición de estructura adoptada por Bourbaki en ese primer volumen puede que sea totalmente rigurosa, pero es además muy intrincada y poco clarificadora, con lo que su utilización resulta en la práctica demasiado engorrosa para servir al propósito de clarificación que se había impuesto (Corry, 1996). Es lo que concluyen diferentes autores (Mac Lane, 1996; Cartier, 1998) al analizar el trabajo de Bourbaki: la presentación bourbakista de las matemáticas como una teoría de estructuras matemáticas carece de esa teoría abstracta de estructuras. Apuntan, sin embargo, como indicábamos anteriormente, a la posibilidad de que la teoría de categorías sirviera, en realidad, para situar de forma natural a las funciones definidas entre estructuras en el centro de la exposición. No olvidemos que el propio Bourbaki confirió gran importancia al concepto de 'morfismo' a la hora de caracterizar las estructuras y las características propias de las estructuras. Es esta una cuestión que, según las críticas, no queda lo suficientemente clara y explícita desde el punto de vista adoptado.

Por otro lado, es indudable que los integrantes de Bourbaki eran matemáticos de primera línea que, aparte del trabajo que realizaron como miembros de Bourbaki estaban trabajando cada cual en su especialidad. Entre ellos podemos encontrar a Ehresmann o Grothendieck, que hicieron grandes contribuciones al desarrollo y a la emancipación de la teoría de categorías, como disciplina independiente ${ }^{11}$, e incluso a Eilenberg, que es junto

11 Podemos mencionar, como ejemplo, el importante artículo de Groethendieck (1957), en el que se definen las categoría abelianas, en cuyo contexto es posible caracterizar un tipo de estructura, refiriéndose exclusivamente a los functores existentes entre las estructuras, sin tener que hablar de los elementos 
con Mac Lane, el fundador de la teoría con su artículo (Eilenberg, Mac Lane, 1945). Bourbaki conocía muy bien la teoría de categorías e incluso hubo intentos posteriores de incorporarlo a su obra (Mashaal, 2002). Sin embargo, lo cierto es que al final no la adoptó explícitamente.

Cartier (1998) apunta que hubo razones de índole práctica cuando habla de la hipocresía de Bourbaki, que aunque adoptó progresivamente un punto de vista cada vez más ligado a la perspectiva categorial de la matemática, debido, según Cartier, a que ofrecía el marco general adecuado para tratar cuestiones relativas a las estructuras matemáticas y sus relaciones, no entendió conveniente revisar su obra adoptando esta perspectiva como fundamento. Una de las razones que esgrime Cartier para ello está relacionada con la exigencia monolítica de rigor (Cartier, 1998, p. 28) que adoptó Bourbaki a la hora de escribir su obra. Como bien señalaron Eilenberg y Mac Lane en el artículo fundacional de la teoría de categorías, existían problemas lógicos a la hora de definir el concepto de categoría dentro de, por ejemplo, la teoría axiomática de conjuntos de Zermelo, ya que hacía falta considerar clases de objetos de cuya condición de conjunto se derivaban inexorablemente paradojas relacionadas con la de Russell. Hubo entonces diferentes intentos de encontrar una teoría axiomática de conjuntos adecuada (probaron, por ejemplo, con la de Gödel-von Neumann, que diferencia entre clases y conjuntos), pero desistieron a la vista de que no hallaban una solución satisfactoria, y decidieron formular la teoría de categorías sin entrar a fondo en cuestiones de fundamentación, es decir, desde una perspectiva más práctica e intuitiva que rigurosa. Bourbaki también se interesó por la fundamentación conjuntista de la teoría de categorías. Entre las soluciones que se discutieron se encuentra, por ejemplo, la que propuso Grothendieck valiéndose del concepto de universo (de Grothendieck). La propuesta consistía en añadir un nuevo axioma, llamado axioma de los universos, al sistema ZFC, que afirmaba que todo conjunto estaba contenido en un universo, y que equivalía a postular una sucesión infinita de cardinales fuertemente inaccesibles. De esta forma se obtenía un sistema conjuntista más potente. Sin embargo, Bourbaki esgrimió el argumento de la falta de seguridad que implicaba adjuntar este axioma a $\mathrm{ZFC}^{12}$ para descartar la vía propuesta por Grothendieck (Krömer, 2006). Para Cartier (que también era miembro de Bourbaki) la renuncia 'filosófica' de Bourbaki, en nombre del rigor absoluto, es ciertamente inaceptable. Según él, de la misma forma que los matemáticos del siglo XVIII aceptaron utilizar los infinitésimos, Bourbaki debería haber aceptado las dificultades lógicas que acarreaba la adopción de la teoría de categorías como 'metateoría' de su obra fundamental, lo cual habría provisto de una mayor coherencia y simplicidad en varios aspectos a la obra de Bourbaki; razón última de la misma, no lo olvidemos.

Todas estas críticas parecerían aceptables, y más teniendo en cuenta su procedencia: al fin y al cabo parece ser que el debate sobre la adopción explícita de la teoría de categorías estuvo sobre la mesa en más de una reunión de Bourbaki, y más de uno de sus integrantes re-

que componían la estructura. Esta es una de las razones por la cual muchos categoristas sostienen que las categorías ofrecen una forma más natural de hablar sobre las estructuras, sin tener que definir, artificialmente, los elementos que las componen.

${ }^{12}$ Nos referimos, en concreto, al hecho de que cuando se debatió este punto parecía haber dificultades con respecto a la consistencia relativa del axioma de los universos. Más adelante se confirmaría este punto con la demostración de la indemostrabilidad de dicha consistencia relativa, pero cuando se dio el debate en su seno, Bourbaki no contaba con este resultado. 
conocía la superioridad de esta teoría con respecto a cualquier otra a la hora de tratar con las estructuras matemáticas (Dieudonné, 1970). Hoy en día, además, no parece haber dudas en que la teoría de categorías es una de las mejores formulaciones de una teoría abstracta de estructuras matemáticas que se haya desarrollado hasta el momento (Corry, 1996; Mac Lane, 1996). En cambio, no estamos de ninguna forma seguros de los beneficios que habría obtenido Bourbaki, de haber adoptado la teoría de categorías y reformulado su trabajo, en una forma más abstracta, si cabe, según se lo pidieron algunos de sus integrantes (Cartier, Dieudonné) y otros que no lo eran (Mac Lane). Al fin y al cabo, somos de la opinión de que se le podría hacer una crítica semejante al punto de vista categorial de las matemáticas, que tantos adeptos tiene entre los que trabajan en teoría de categorías y sus entornos inmediatos, pero por el contrario, no demasiados fuera de ellos. De la misma forma que algunas teorías se simplificarían y optimizarían adoptando el lenguaje y métodos categoriales (en especial, cómo no, aquellas para las que se creó: topología algebraica, geometría diferencial abstracta, etc.), otras quedarían fuera de su alcance. Es indudable que hay muchas áreas que no ofrecen resquicios por donde introducir de forma coherente la maquinaria categorial: hablamos de muchas áreas del análisis, de la algorítmica y de la combinatoria entre otras ${ }^{13}$.

De todos modos, cabe señalar que esta es una vía que ha ido materializándose en lo que se ha venido a llamar 'estructuralismo categorial' en fundamentos de las matemáticas. Podríamos encontrar a dos promotores de esta vía en (Lawvere, 1964; Lawvere, 1966) y (Awodey, 1996; Awodey, 2004), abogando por la supremacía del punto de vista categorial de las matemáticas, aunque sea desde perspectivas ciertamente diferenciadas. El primero propone un topos como fundamento para las matemáticas; el segundo niega que las matemáticas necesiten ningún fundamento, entendido la fundamentación en el sentido clásico, y opta por una reorganización estructural de las matemáticas guiada por la teoría de categorías ${ }^{14}$.

Parece ser por tanto que el problema radica en querer desarrollar las teorías sobre un único concepto fundamental. Y no solo eso, además, sobre una única formalización de ese concepto fundamental, ya sea el de estructura conjuntista (Bourbaki), ya el de estructura caracterizada por flechas y functores (categorías). Al fin y al cabo no cabe duda de que cada formalización se adapta mejor a aquellas teorías que son más cercanas al ámbito en el que fueron originadas. $\mathrm{Y}$ a su vez resultan altamente artificiales en aquellas áreas en las que si existiese alguna forma de adoptarlas como fundamento, sería a costa de la pérdida de la coherencia y simplicidad de las teorías. Actuación, claro está, que se opone frontalmente al objetivo perseguido.

${ }^{13}$ Se puede profundizar mucho más de lo que aquí hemos hecho en la relación que existió entre el trabajo de Bourbaki y el desarrollo de la teoría de categorías. Ciertamente ambos procesos coincidieron en el tiempo, y compartieron algunos de sus protagonistas principales. Quisieramos simplemente señalar dos importantes referencias en este sentido. Por un lado (Corry, 1996) estudia en su última parte tres diferentes teorías de estructuras abstractas que se han propuesto durante el siglo $\mathrm{xx}$, entre las cuales la teoría de categorias es la que sale mejor posicionada como posible marco para desarrollar una perspectiva estructuralista de la matemática como la que quiso ofrecer Bourbaki. Por otro lado (Krömer, 2007), es un trabajo dedicado exclusivamente a estudiar la historia de la teoría de categorías y sus implicaciones para la filosofía de las matemáticas, en las que se recogen muchos de los aspectos relacionados con el punto de vista de Bourbaki con respecto a diferentes cuestiones de la teoría de categorías.

14 Para tener una perspectiva global del estructuralismo categorial podríamos remitir al lector a (Landry, Marquis, 2005). 
Utilizando la expresión de Demazure, Bourbaki fue víctima de la ilusión fundamental de que para cualquier cuestión matemática existía un único punto de vista con el que abordarlo (Mashaal, 2002). Esta misma crítica serviría, según lo que hemos expuesto, para rebatir la posición de que el trabajo de Bourbaki hubiese logrado una mayor coherencia y alcance si hubiese adoptado como teoría marco la teoría de categorías. Desde el punto de vista actual es insostenible que una única formalización como la teoría de conjuntos o la teoría de categorías se pueda considerar como el verdadero fundamento, sobre el cual se sostendría el edificio deductivo de las matemáticas. Es imposible encontrar un único marco que se pueda usar en todos los casos. Si había dudas en los tiempos en que Bourbaki tuvo mayor influencia, hoy en día estas dudas se han disipado. Y por tanto, la tan mencionada y tan asumida metáfora del 'edificio matemático' no se puede considerar en adelante una metáfora válida para describir cuál es el panorama de las matemáticas de hoy en día. No es posible dar una axiomatización global de la matemática en la forma en que hizo Hilbert en las Grundlagen con la geometría euclídea (Hilbert, 1899). Sin embargo, creo que es posible hacer una nueva lectura de la metáfora de la 'ciudad matemática' que proponía Bourbaki en su 'manifiesto' (1950) y a la que aludíamos al principio de este artículo.

Los conjuntos, las funciones y las estructuras son conceptos básicos dentro de la matemática, pero no en el sentido rígido en el que se han utilizado hasta ahora, es decir, haciendo referencia a una única formalización, óptima, para cada uno de ellos y centrando el debate en cuál de ellos es previo a cual y en qué sentido (Feferman, 1977). Acabamos de concluir que esa vía está agotada. Diferentes formalizaciones de una misma noción general pueden ser igualmente válidas, cada una en un terreno distinto. Es lo que ocurre con las estructuras, la noción de estructura no tiene por qué apuntar necesariamente a estructuras conjuntistas como las utilizadas por Bourbaki, ni a la teoría de categorías. Lo que no es óbice para que tanto unas como otras sean representaciones legítimas, incluso, óptimas, en ciertos terrenos de las matemáticas, normalmente porque han surgido precisamente en esos contextos. Es por ello por lo que pensamos que en el caso de que sea posible dar criterios organizativos que den cuenta de la unidad de las matemáticas de hoy en día, como hizo Bourbaki, debemos adoptar criterios más flexibles. Nociones generales más que formalizaciones concretas de estas nociones como pueden ser las nociones generales de operación o función, clase o conjunto y estructura. Bourbaki nos enseñó cómo las estructuras desempeñan un papel fundamental en la organización coherente de las matemáticas. En lo que no estamos tan de acuerdo, es en que éstas constituyan el principio ordenador de las matemáticas. Y este era precisamente el lema de Bourbaki.

Existe un componente que, teniendo para Bourbaki un papel importante en su reorganización, en su obra no se le asigna el papel central que creemos que desempeña en la organización interna de las matemáticas ${ }^{15}$ :

Pero la noción de 'morfismo' importa. Bourbaki y muchos otros siempre establecían una cuestión central sobre cualquier nuevo artilugio matemático: '¿Cuáles son los morfismos?' La estructura conduce inevitablemente al morfismo para Bourbaki y para todos los demás.

15 But the notion of 'morphism' matters. Bourbaki and many others always put a central question to any new mathematical gadget: 'What are the morphisms?' Structure inevitably leads to morphisms for Bourbaki and everyone else (Mac Lane, 1996, p. 181). Traduccion del autor. 
Pensamos que más allá de las estructuras, es la noción general de morfismo, o de función, la que mejor caracteriza la naturaleza de las matemáticas de hoy en día (Ezenarro, 2013). Históricamente se ha podido observar que se han dado reorganizaciones fundamentales en las matemáticas cuando se ha vuelto la atención sobre las funciones significativas de cada teoría, y las estructuras que forman éstas. Son estructuras de funciones relevantes, por ejemplo, los grupos formados por ciertas permutaciones específicas de las raíces de una ecuación polinómica, con respecto a la operación de composición de permutaciones, que consideró Galois para hallar la solución última a su problema. Otro tanto podríamos decir de la clasificación de los sistemas geométricos obtenido por Klein centrándose en los grupos de simetrías de cada sistema geométrico. ¿Qué son las simetrías sino las funciones significativas, que dejan invariantes las propiedades intrínsecas de la correspondiente geometría? Esas son las funciones cuyas estructuras hay que delimitar para poder clarificar debidamente la teoría en cuestión. Algo similar sucedió también con el álgebra lineal. Los cálculos matriciales carecían de la motivación suficiente antes de la definición axiomática de espacios vectoriales y sus correspondientes morfismos (que no eran más que las transformaciones lineales). La aparición de los conceptos de espacio vectorial y de las transformaciones lineales sirvió para aclarar la situación: las matrices no eran más que representaciones de las transformaciones lineales. Incluso nos parece que puede haber evidencias a favor de que algo de este tipo podría darse con la teoría de homotopía y la clasificación de los espacios topológicos. Los grupos de homotopía de los espacios topológicos también pueden ser considerados como estructuras formadas por las funciones que son significativas en este caso y que no son otras que clases de equivalencia de lazos basados en un punto del espacio. Como se puede observar, son todos ellos ejemplos que cabrían dentro de lo que llamamos noción general de función.

Pero no solo tenemos el papel decisivo que juegan las estructuras de funciones significativas de una teoría a la hora de hallar reformulaciones efectivas de esa teoría, sino que, también cabría bajo esa misma denominación general, otro aspecto que se observa de forma creciente en diferentes partes de las matemáticas, casi siempre unidos a grandes avances en la resolución de problemas importantes, como lo es sin duda la interacción functorial entre diferentes partes de las matemáticas. La efectividad que se deriva de la posibilidad de definir estructuras de un tipo o de otro en una teoría concreta, es una evidencia de la naturaleza functorial (y no solo categorial) de las matemáticas. Encontrar una estructura relevante en un área, cuando la obtención de nuevos resultados resulta costosa, permite volcar toda la teoría de esa estructura específica sobre el área en cuestión, de forma que se puedan resolver problemas que se encontraban abiertos e incluso abrir nuevas vías de investigación. Encontramos un claro ejemplo de esta afirmación en el área dónde también encuentra su origen la teoría de categorías y functores, que no es otra que la topología algebraica. Diferentes áreas se encuentran interconectadas functorialmente en matemáticas y, precisamente, en la construcción de estas interconexiones podemos encontrar una característica fundamental del quehacer matemático actual. Podríamos encontrar en esa interacción que existe entre las diferentes estructuras matemáticas, una posible explicación de la unidad que existe entre partes importantes de las matemáticas ${ }^{16}$. Bajo esta perspectiva podríamos describir la matemática, no como un edificio, sino, más bien, como un 'grafo' de estructuras interconectadas

16 No nos atrevemos a ir más allá, puesto que no está claro que ésta sea una característica generalizable a todas las áreas. Puede que existan áreas, ciertamente inconexas dentro del entramado matemático. 
functorialmente, a través de los cuales se establecen flujos de información. Encontraríamos en este 'grafo' un nuevo principio organizativo, que según nuestro punto de vista se adecua mejor a la situación actual. Es este nuevo punto de vista en la fundamentación interna de las matemáticas a lo que llamamos funcionalismo estructuralista (Ezenarro, 2013).

Cabe mencionar que el 'grafo' de estructuras parece evocar de alguna forma la metáfora de la matemática como ciudad de la que hablábamos: la ciudad (la matemática) como una multiplicidad de barrios (estructuras) conectados por calles y avenidas (functores, funciones), que sirven para establecer flujos (de información) entre diferentes barrios (estructuras). Como se puede observar la analogía, aunque sugerente, resulta cuanto menos difusa. Pienso que el concepto matemático de grafo resulta más significativo que el de ciudad, en tanto en cuanto lo despoja de cualidades superfluas. Sin olvidar que el entrecomillado sirve para resaltar que el término se utiliza en sentido figurado, y que en ningún momento se sugiere utilizar la teoría matemática de grafos como herramienta metamatemática para modelar el propio funcionamiento de la matemática.

\section{Conclusiones}

Nos parece que el trabajo de Bourbaki cumple una función de forma clara: la de poner en primera línea el concepto general (y no un formalismo concreto), en su tiempo emergente, de estructura matemática, y tratar de reorganizar la matemática bajo la asunción, si se quiere, 'filosófica', de que en ella se encuentra el verdadero principio organizador de las matemáticas. Con teoría de estructuras o sin ella, es indudable que es ésta una tesis clara que emana de la monumental obra de Bourbaki. Y no es menos cierto, que desde la perspectiva de la matemática actual, en la que se puede observar claramente la gran explosión de métodos y teorías de diferente índole, que abarcan un gran número de formas de afrontar los diferentes retos, el enfoque bourbakista no se adapta bien a ramas enteras de las matemáticas. Bourbaki obtuvo, sin embargo, victorias parciales muy importantes, como por ejemplo es el caso de la topología y el análisis funcional, además de diferentes teorías algebraicas, como el álgebra conmutativa o los grupos de Lie, que era, en definitiva, el espacio natural de donde provenía la empresa organizativa de Bourbaki, como hemos visto anteriormente.

\section{REFERENCIAS}

Anacona, Maribel, Luis C. Arboleda, y Francisco J. Pérez-Fernández. 2014. On Bourbaki's Axiomatic System for Set Theory. Synthese 191: 4069-4098.

Awodey, Steve. 1996. Structure in Mathematics and Logic: A Categorical Perspective. Philosophia Mathematica 4(3): 209-237.

Awodey, Steve. 2004. An Answer to Hellman's Question: 'Does Category Theory Provide a Framework for Mathematical Structuralism?' Philosophia Mathematica 12(1): 54-64.

Bourbaki, Nicolas. 1939-. Éléments de mathématique. Paris: Hermann.

Bourbaki, Nicolas. 1950. The Architecture of Mathematics. American Mathematical Monthly 57(4): 221232.

Cartier, Pierre. 1998. Notes sur l'histoire et la philosophie des mathématiques, III. Le structuralisme en mathématiques: mythe ou réalité? Paris: Preprints IHES.

Corry, Leo. 1996. Modern algebra and the rise of mathematical structures. Basel-Boston-Berlin: Birkhäusser.

Davis, Philip J. y Reuben Hersh. 1981. The Mathematical Experience. Boston: Birkhäuser. 
Dieudonné, Jean. 1970. The Work of Nicolas Bourbaki. American Mathematical Monthly 77: 134-145.

Dieudonné, Jean. 1979. The Difficult Birth of Mathematical Structures. (1840-1940). En Scientific Culture in Contemporary World editado por Vittorio Mathieu y Paolo Rossi, 7-23. Milan: Scientia.

Eilenberg, Samuel, y Saunders Mac Lane. 1945. General Theory of Natural Equivalences. Transactions of the American Mathematical Society 28: 231-294.

Ezenarro, Enetz. 2013. Estrukturalismotik Funtzionalismora matematikaren barne oinarrietan. Tesis doctoral inédita. Universidad del País Vasco.

Feferman, Solomon. 1977. Categorical Foundations and Foundations of Category Theory. En Logic, Foundations of Mathematics and Computability Theory editado por Robert E. Butts y Jaakko Hintikka, 149169. Reidel: Dordrecht.

Ferreirós, José, y Jeremy Gray (eds.). 2006. The Architecture of Modern Mathematics. Essays in History and Philosophy. Oxford: Oxford University Press.

Groethendieck, Alexander. 1957. Sur quelques points d'algèbre homologique, I. Tohoku Mathematical Journal 9(2): 119-121.

Hermann, Robert. 1986. Mathematics and Bourbaki. The Mathematical Intelligencer 8(1): 32-33.

Hernández, Jesús. 2002. La matemática y sus elementos: de Euclides a Bourbaki. La Gaceta de la Real Sociedad Matemática Española 5(3): 649-672.

Hilbert, David. 1899. Grundlagen der Geometrie. Leipzig: Teubner.

Klein, Felix. 1872. Vergleichende Betrachtungen über neuere geometrische Forschungen. Erlangen.

Kneebone, Geoffrey T. 1963. Mathematical Logic and the Foundations of Mathematics. London: Van Nostrand.

Krömer, Ralf. 2006. La "machine de Grothendieck", se fonde-t-elle seulement sur des vocables métamathématiques? Bourbaki et les catégories au cours des années cinquante. Revue d'Histoire des Mathématiques 12: 111-154.

Krömer, Ralf. 2007. Tool and Object. A History and Philosophy of Category Theory. Basel-Boston-Berlin: Birkhäusser.

Landry, Elaine, y Jean P. Marquis. 2005. Categories in Context: Historical, Foundational and Philosophical. Philosophia Mathematica 13(3): 1-43.

Lawvere, Francis W. 1964. An elementary theory of the Category of Sets. Proceedings of the National Academy of Science of the U.S.A. 52: 1506-1511.

Lawvere, Francis W. 1966. The Category of Categories as a Foundation for Mathematics. En Proceedings of the Conference on Categorical Algebra editado por Samuel Eilenberg, David K. Harrison, Helmut Röhrl y Saunders Mac Lane, 1-20. Berlin-Heidelberg: Springer-Verlag.

Mac Lane, Saunders. 1986. Mathematics: Form and Function. New York: Springer-Verlag.

Mac Lane, Saunders. 1996. Structure in Mathematics. Philosophia Mathematica 4(2): 174-183.

Mashaal, Maurice. 2002. Bourbaki: Une société secrète de mathématiciens. Paris: Éditions Pour la Science.

Schwartz, Laurent. 1997. Un mathematicien aux prises avec le siècle. Paris: Odile Jacob.

Sharpe, Richard W. 1997. Differential Geometry. Cartan's Generalization of Klein's Erlangen Program. New York: Springer.

van der Waerden, Bartel L. 1930-1931. Moderne Algebra. Berlin: Springer.

Wussing, Hans. 1969. Die Genesis des abstrakten Gruppenbegriffes. Berlin: VEB Deutscher Verlag der Wissenschaften.

Enetz Ezenarro Arriola es licenciado en matemáticas y doctor en ciencias cognitivas y humanidades. Es profesor de didáctica de las matemáticas en la Universidad del País Vasco. Sus principales líneas de trabajo son los fundamentos de las matemáticas y el análisis histórico con aplicaciones a la educación matemática.

Dirección: Instituto de Lógica, Cognición, Lenguaje e Información (ILCLI) y Escuela de Magisterio de Bilbao. Universidad del País Vasco/ Euskal Herriko Unibertsitatea. Departamento de Didáctica de las Matemáticas y de las Ciencias Experimentales. Barrio Sarriena, s/n. 48940 Leioa, Bizkaia. E-mail: enetz.ezenarro@ehu.eus 